\title{
HERMITE CONJUGATE EXPANSIONS
}

\author{
BY \\ BENJAMIN MUCKENHOUPT( $\left.{ }^{1}\right)$
}

1. Introduction. Professor E. M. Stein suggested that conjugate function theorems such as those proved in [3] for Gegenbauer expansions or the well-known ones for Fourier expansions would have analogues in the theory of Hermite and Laguerre expansions. It is shown in this paper that this is indeed the case for Hermite expansions. As in the other cases, it is proved here that the mapping from a function to its conjugate function or its conjugate Poisson integral is a bounded operator in $L^{p}\left(\exp \left(-x^{2}\right)\right)$ for $1<p<\infty$. Furthermore, the conjugate Poisson integral converges in $L^{p}$ norm and pointwise almost everywhere to the conjugate function. Substitute results for the case $p=1$ are also proved. This leads to the possibility of an $H^{p}$ theory for Hermite expansions and analogues of the classical applications of ordinary conjugate functions.

If $f(y)$ is in $L^{1}\left(\exp \left(-y^{2}\right)\right)$, the Poisson integral, $f(x, y)$, will be defined as in [2] and an integral definition of the conjugate Poisson integral, $f^{f}(x, y)$, will be given for $x>0$. If $f(y)$ has Hermite expansion $\sum a_{n} H_{n}(y)$, then for each $x>0 f(x, y)$ has Hermite expansion $\sum a_{n} \exp \left(-(2 n)^{1 / 2} x\right) H_{n}(y)$ and $f(x, y)$ will be shown to have Hermite expansion $\sum a_{n}(2 n)^{1 / 2} \exp \left(-(2 n)^{1 / 2} x\right) H_{n-1}(y)$. As mentioned in [2], merely defining the functions to be the sum of these series is unsatisfactory since the series may diverge for all $x$ and $y$ for some $f(y)$ in $L^{p}\left(\exp \left(-y^{2}\right)\right), 1 \leqq p<2$. The definitions used will be meaningful for any $f(y)$ in $L^{1}\left(\exp \left(-y^{2}\right)\right)$.

The conjugate function, $f(y)$, will be defined as $\lim _{x \rightarrow 0^{+}} f(x, y)$. It will be shown that if $f$ is in $L^{1}\left(\exp \left(-y^{2}\right)\right)$, then this limit exists for almost every $y$. If $f$ has the Hermite expansion given above, then $f(y)$ will be shown to have the expansion $\sum a_{n}(2 n)^{1 / 2} H_{n-1}(y)$.

It was shown in [2] that

$$
\frac{\partial^{2} f(x, y)}{\partial x^{2}}+\exp \left(y^{2}\right) \frac{\partial}{\partial y}\left(\exp \left(-y^{2}\right) \frac{\partial f(x, y)}{\partial y}\right)=0
$$

Similarly, it will be shown here that

$$
\frac{\partial^{2} f^{x}(x, y)}{\partial x^{2}}+\frac{\partial}{\partial y}\left[\exp \left(y^{2}\right) \frac{\partial}{\partial y}\left(\exp \left(-y^{2}\right) f(x, y)\right)\right]=0
$$

and that the analogues of the Cauchy-Riemann equations

$$
\frac{\partial f(x, y)}{\partial x}=\exp \left(y^{2}\right) \frac{\partial}{\partial y}\left(\exp \left(-y^{2}\right) f(x, y)\right)
$$

Received by the editors August 11, 1967 and, in revised form, March 14, 1968.

(1) Supported in part by NSF Grant GP4219. 
and

$$
\frac{\partial f(x, y)}{\partial y}=-\frac{\partial}{\partial x} \tilde{f}(x, y)
$$

are valid for $x>0$. Equations (1.1) and (1.2) would look more alike if $\exp \left(-y^{2}\right) f(x, y)$ were taken as the conjugate integral. This procedure was used at the corresponding point in [3] but $f(x, y)$ seems more natural since the norm inequalities are true for it.

As was the case in [2], a simple imitation of the methods used in [3] produces extreme complications. Consequently, two modifications of classical Hilbert transform theorems are proved in $\$ 2$. The first uses a simple method to produce an estimate for integrals with variable kernels that bear some resemblance to the Hilbert transform. The estimate is in terms of a sup of singular integrals. For the transform $\int f(z) K(y, z) d z$, the estimate is valid if $K(y, y+h)=-K(y, y-h)$ and for each $y, h K(y, y+h)$ has total variation bounded by a constant independent of $y$. This is easy to use since, in the case considered, $K(y, z)$ is differentiable in $z$ and it is enough to verify that the integral of the absolute value of the derivative of $h K(y, y+h)$ is bounded.

The second result in $\S 2$ shows that if $c$ is sufficiently small, then

$$
\sup _{0<a<b<c}\left|\int_{a<|t|<b} \frac{f(y+t)}{t} d t\right|
$$

will satisfy a norm inequality for measures other than $d y$. This is a simple consequence of the classical theory.

In $\S 3$ the definition of $\tilde{f}(x, y)$ as an integral is stated and the simple consequences of the definition are given. Next the basic lemma, Lemma 4, is stated; it asserts that the kernel for $\tilde{f}(x, y)$ can be written as the sum of two parts with specific properties. The proof involves careful estimation of various integrals that arise; the details are given in $\$ \$ 4-6$. The two parts are chosen so that a theorem in [2] can be applied to one and the first lemma in $\$ 2$ to the other.

In $\S 7$ use is made of Lemma 4 to show that $f(x, y)$ is dominated by a Hardy max function plus a sup of singular integrals. This estimate is used to prove norm inequalities and that as $x \rightarrow 0^{+}, \tilde{f}(x, y)$ converges almost everywhere and in norm provided $f(y)$ is in $L^{p}\left(e^{-y^{2}}\right)$ for $p>1$. Here use is made of the second lemma in $\S 2$ and a result concerning Hardy maximal functions in [2]. A substitute result is also proved for the case when $p=1$.

2. Singular integral lemmas. In this section two general results are proved that are useful for treating the conjugate Poisson integrals for Hermite expansions. The first, Lemma 1 , is concerned with kernels that are not precisely $1 / z$ but bear some resemblance to them. This result was mentioned to me orally by Professor B. F. Jones; since it is unpublished and simple, a proof of it is included here for completeness. 
LEMMA 1. If $K(z)=-K(-z)$ and if $z K(z)$, (defined as 0 for $z=0$ ), has total variation $V$ on $[0, m]$, then

$$
\sup _{0<a<b<m}\left|\int_{a \leqq|z| \leqq b} f(y-z) K(z) d z\right| \leqq V \sup _{0<a<b<m}\left|\int_{a \leqq|z| \leqq b} \frac{f(y-z)}{z} d z\right| .
$$

Because of the assumption on the variation of $z K(z)$, there is a signed measure, $\mu$, of bounded variation such that $z K(z)=\int_{0}^{z} d \mu(t)$ for almost every $z$ with $0 \leqq z \leqq m$. Then if $0<a<b \leqq m$, the fact that $K$ is odd shows that

$$
\begin{aligned}
\int_{a \leqq|z| \leqq b} f(y-z) K(z) d z & =\int_{a}^{b} \frac{f(y-z)-f(y+z)}{z} z K(z) d z \\
& =\int_{a}^{b} \frac{f(y-z)-f(y+z)}{z}\left(\int_{0}^{z} d \mu(t)\right) d z .
\end{aligned}
$$

Interchanging the order of integration shows that this equals

$$
\int_{0}^{b}\left(\int_{\max (t, a)}^{b} \frac{f(y-z)-f(y+z)}{z} d z\right) d \mu(t) .
$$

The absolute value of this last expression, however, is clearly less than or equal to the right side of the inequality in the lemma.

To obtain a norm inequality with a measure other than $d y$ for an ordinary singular integral, two restrictions will be imposed. The measure considered will be one that can be written as a weight function, $w(y)$, times $d y$. The range of the singular integral must be restricted so that $w(y)$ does not change drastically. The details are specified in the definition of property $A$ below. Lemmas 2 and 3 then are automatic. Fortunately this approach is sufficient for the integral that arises.

Given a positive weight function $w(y)$ on an interval, $I$, a partition of $I$ into closed subintervals will be said to have property $A$ with multiplier $B$ if:

1. A compact subset of $I$ not containing an endpoint of $I$ intersects a finite number of the subintervals.

2. An interval of the partition is no more than twice as long as the adjacent intervals.

3. The ratio of $\sup w(y)$ to inf $w(y)$ on an interval is no more than $B$.

Note that because of condition 1 , intervals in such a partition can be indexed with the integers in their natural order.

LEMMA 2. Let $w(y)$ be a positive function, $f(y)$ be in $L^{p}[w(y) d y], 1<p<\infty$, on an interval $I$, and $\left\{I_{n}\right\}$ a partition of I having property $A$ with multiplier $B$. Define

$$
g(y)=\sup \left|\int_{a \leqq|z| \leqq b} \frac{f(y-z)}{z} d z\right|
$$

where the sup is taken over all $a$ and $b$ such that $0<a<b$ and $b$ is not greater than half 
the length of the $I_{n}$ in which $y$ lies. Then there exists a constant, $C$, depending only on $p$ and $B$ such that

$$
\int_{I}|g(y)|^{p} w(y) d y \leqq C \int_{I}|f(y)|^{p} w(y) d y .
$$

Let $I_{n}=\left[y_{n}, y_{n+1}\right]$. Then

$$
\int_{I}|g(y)|^{p} w(y) d y=\sum_{n} \int_{I_{n}}|g(y)|^{p} w(y) d y \leqq \sum_{n} B w\left(y_{n}\right) \int_{I_{n}}|g(y)|^{p} d y
$$

by use of condition 3. For a particular $I_{n}$ the same $g$ would result if $f$ were 0 outside $J_{n}=I_{n-1} \cup I_{n} \cup I_{n+1}$ because of condition 2 . The usual theorem on the sup of the Hilbert transform, $\left[1\right.$, p. 116], then shows that there is a constant, $A_{p}$, depending only on $p$ such that $\int_{I_{n}}(g(y))^{p} d y \leqq A_{p} \int_{J_{n}}|f(y)|^{p} d y$. Using this in the preceding inequality then shows that

$$
\int_{I}(g(y))^{p} w(y) d y \leqq \sum_{n} A_{p} B w\left(y_{n}\right) \int_{J_{n}}|f(y)|^{p} d y \leqq \sum_{n} A_{p} B^{3} \int_{J_{n}}|f(y)|^{p} w(y) d y .
$$

This is less than or equal to $3 A_{p} B^{3} \int_{I}|f(y)|^{p} w(y) d y$ as desired.

A substitute result for $p=1$ may be stated as follows.

LEMMA 3. Let $w(y)$ be a positive function and let $f(y)$ be in $L^{1}[w(y) d y]$ on an interval I. Let $\left\{I_{n}\right\}$ and $g$ be as in Lemma 2, and let $E_{a}=\{y \mid g(y)>a\}$. Then there is a constant $C$, depending only on $B$ such that

$$
\int_{E_{a}} w(y) d y \leqq \frac{C}{a} \int_{I}|f(y)| w(y) d y .
$$

The proof is similar to the proof of Lemma 2. First, observe that

$$
\int_{E_{a}} w(y) d y=\sum_{n} \int_{E_{a} \cap I_{n}} w(y) d y \leqq \sum_{n} B w\left(y_{n}\right) m\left(E_{a} \cap I_{n}\right) .
$$

Now using Theorem 2 on p. 152 of [4], the fact that the Hilbert transform is of weak type $(1,1)$ and the fact that $E_{a} \cap I_{n}$ depends only on the values of $f(y)$ in $J_{n}$ shows that $m\left(E_{a} \cap I_{n}\right) \leqq(C / a) \int_{J_{n}}|f(y)| d y$. The rest of the proof is the same as the last part of the proof of Lemma 2.

3. The conjugate Poisson integral. If $f(y)$ is in $L^{1}\left(\exp \left(-y^{2}\right)\right)$ on $(-\infty, \infty)$, the Poisson integral, $f(x, y)$, will be defined as it was in (3.11) of [2] as

$$
f(x, y)=\int_{-\infty}^{\infty} R(x, y, z) f(z) \exp \left(-z^{2}\right) d z
$$

where

$$
R(x, y, z)=\int_{0}^{1} \frac{x \exp \left(x^{2} / 2 \log r\right)}{\pi r\left(2\left(1-r^{2}\right)\right)^{1 / 2}(-\log r)^{3 / 2}} \exp \left(\frac{-r^{2} y^{2}+2 r y z-r^{2} z^{2}}{1-r^{2}}\right) d r
$$


By Corollary 3 of [2], if $f(y)$ has Hermite expansion $\sum a_{n} H_{n}(y)$, then for each $x>0$ $f(x, y)$ has the expansion $\sum a_{n} \exp \left(-(2 n)^{1 / 2} x\right) H_{n}(y)$. Consequently,

$$
R(x, y, z)=\sum_{n=0}^{\infty} \frac{\exp \left(-(2 n)^{1 / 2} x\right) H_{n}(y) H_{n}(z)}{\pi^{1 / 2} 2^{n} n !}
$$

To make the conjugate Poisson integral, $f(x, y)$, satisfy (1.4), it is clear that it should be defined by

$$
f(x, y)=\int_{-\infty}^{\infty} Q(x, y, z) f(z) \exp \left(-z^{2}\right) d z
$$

where

$$
Q(x, y, z)=\int_{0}^{1} \frac{2^{1 / 2}(z-r y) \exp \left(x^{2} / 2 \log r\right)}{\pi(-\log r)^{1 / 2}\left(1-r^{2}\right)^{3 / 2}} \exp \left(\frac{-r^{2} y^{2}+2 r y z-r^{2} z^{2}}{1-r^{2}}\right) d r .
$$

Expression (3.5) is obtained from (3.2) by differentiating with respect to $y$, integrating with respect to $x$, using the fact that $Q$ should approach 0 when $x$ approaches $\infty$, and multiplying by -1 . The basic facts about this definition are as follows.

THEOREM 1. For every $x>0$ and every $y, Q(x, y, z)$ is a bounded function of $z$. If $f(y)$ is in $L^{1}\left(\exp \left(-y^{2}\right)\right)$, then $f(x, y)$ exists for every $x>0$ and every $y$, and (1.2)(1.4) are valid. If $f(y)$ has Hermite expansion $\sum a_{n} H_{n}(y)$, then for each $x>0 f(x, y)$ has the Hermite expansion

$$
\sum_{n=1}^{\infty} a_{n} \exp \left(-(2 n)^{1 / 2} x\right)(2 n)^{1 / 2} H_{n-1}(y)
$$

For every $p<2$ there exists an $f(y)$ in $L^{p}\left(\exp \left(-y^{2}\right)\right)$ such that for every $x$ (3.6) diverges for every $y$. If $x>0$ and $f(y)$ is in $L^{2}\left(\exp \left(-y^{2}\right)\right)$, then (3.6) converges to $\tilde{f}(x, y)$ for almost every $y$.

By considering separately $0 \leqq r<\frac{1}{2}$ and $\frac{1}{2} \leqq r<1$ and replacing $-\log r$ by a suitable multiple of $1-r$ in the second case, it is clear that

$$
\frac{\exp \left(x^{2} / 2 \log r\right)}{(-\log r)^{1 / 2}\left(1-r^{2}\right)^{3 / 2}} \leqq C\left(1+1 / x^{4}\right), \quad 0 \leqq r<1 .
$$

Here and hereafter $C$ will denote a constant not necessarily the same at each occurrence. Using (3.7) in (3.5) and multiplying and dividing by $\exp \left(y^{2}\right)$ shows that

$$
|Q(x, y, z)| \leqq C\left(1+x^{-4}\right) \exp \left(y^{2}\right) \int_{0}^{1}|z-r y| \exp \left(-(y-r z)^{2} /\left(1-r^{2}\right)\right) d r .
$$

If $|z|<|y|$, the integral in (3.8) is bounded by a constant times $|y|$. If $|z| \geqq|y|$, replace the integrand with $2|z| \exp \left[-(y-r z)^{2}\right]$ which is larger; this shows that the integral in (3.8) is bounded by $2 \pi^{1 / 2}$. This proves the first assertion in the theorem. 
The existence of $f(x, y)$ follows immediately from the first part. Differentiating under the integral sign to obtain the first terms in (1.3) and (1.4) is justified since it was shown in the proof of [2, Corollary 3] that the differentiated kernels are bounded functions of $z$ and $x$ for $x \geqq a>0$. The differentiated kernels for the right sides are the same, justifying both the differentiation under the integral sign and the equalities. In the case of (1.4) it is immediate that the kernels are the same; for (1.3) the kernel of one side must be integrated by parts to make it look like the other.

The first term in (1.2) can be obtained by differentiating under the integral sign since the kernels for $\partial \tilde{f}(x, y) / \partial x$ and $\partial^{2} \tilde{f}(x, y) / \partial x^{2}$ are both bounded. This boundedness follows in the same way as $Q$ 's boundedness since an analogy to (3.7) will be true with higher powers of $\log r$ in the denominator. Differentiating under the integral for the first derivative in the second term in (1.2) was justified in the proof of (1.3). Differentiating under the integral sign for the second derivative again follows by integrating the resulting kernel by parts and observing that it is -1 times the kernel for the first term in (1.2). This last fact then completes the proof of (1.2).

Following the same reasoning as was used in [2, Lemma 2], it can be shown that

$$
Q(x, y, z)=\sum_{n=0}^{\infty} \frac{\exp \left(-(2 n)^{1 / 2} x\right)(2 n)^{1 / 2} H_{n-1}(y) H_{n}(z)}{\pi^{1 / 2} 2^{n} n !}
$$

and

$$
\int_{-\infty}^{\infty} Q(x, y, z) H_{n-1}(y) \exp \left(-y^{2}\right) d y=\frac{\exp \left(-(2 n)^{1 / 2} x\right) H_{n}(z)}{(2 n)^{1 / 2}}
$$

Using (3.8) and the substitution $y=r z+w\left(1-r^{2}\right)^{1 / 2}$ and integrating $w$ first shows that for a fixed $n$

$$
\int_{-\infty}^{\infty}\left|H_{n-1}(y) Q(x, y, z) \exp \left(-y^{2}\right)\right| d y \leqq C\left(1+|z|^{n}\right)\left(1+x^{-4}\right)
$$

If $f$ has an Hermite expansion, then $\int_{-\infty}^{\infty}|f(z)|\left(1+|z|^{n}\right) \exp \left(-z^{2}\right) d z$ exists so that $\left|H_{n-1}(y) Q(x, y, z) \exp \left(-y^{2}\right) f(z) \exp \left(-z^{2}\right)\right|$ is integrable as a function of two variables. This justifies the use of Fubini's theorem and (3.10) to prove that (3.6) is the Hermite expansion of $f(x, y)$. The last two assertions are proved in the same way that the corresponding statements in [2, Lemma 2], were proved.

All of the norm inequalities and convergence theorems for $\tilde{f}(x, y)$ depend upon the following lemma.

LEMMA 4. For every $x \geqq 0, Q(x, y, z)$ can be written as the sum of two functions, $J(x, y, z)$ and $K(x, y, z) \exp \left(z^{2}\right)$, with the following properties. $J(x, y, z)$ is less than $a$ function $L(y, z)$ that is monotone increasing in $z$ for $z<y$, monotone decreasing in $z$ for $z>y$ and $\int_{-\infty}^{\infty} L(y, z) \exp \left(-z^{2}\right) d z$ has a bound independent of $y . K(x, y, y+h)$ $=-K(x, y, y-h), K(x, y, y+h)=0 \quad$ if $|h|>\min (1,1 /|y|)$, and $h K(x, y, y+h)$ 
(defined as 0 for $h=0$ ) as a function of $h$ has total variation bounded by a constant independent of $x$ and $y$.

To prove this define $J(x, y, z)$ to be

$$
\frac{Q(x, y, z)+Q(x, y, 2 y-z) \exp (4 y(z-y))}{2}, \quad|y-z|<\min (1,1 /|y|)
$$

and

$$
Q(x, y, z), \quad|y-z| \geqq \min (1,1 /|y|) .
$$

It is sufficient to prove the conclusions of Lemma 4 for $y \geqq 0$ since $Q(x, y, z)$ $=-Q(x,-y,-z)$. It will be shown in $\$ \$ 4$ and 5 that if the constant $C$ is properly chosen, then $|J(x, y, z)| \leqq L(y, z)$ for $x \geqq 0$ and $y \geqq 0$ where for $y>2$

$$
\begin{array}{rlrl}
L(y, z) & =C / y, & z \leqq 0, \\
& =C \exp \left(z^{2}\right) / y, & 0<z \leqq \frac{1}{2} y, \\
& =C \exp \left(z^{2}\right)\left(1 / y+1 / 2\left(y(y-z)^{3}\right)^{1 / 2}\right), & \frac{1}{2} y<z \leqq y-1 / y, \\
& =C y \exp \left(y^{2}\right)(1-\log [y|y-z|]), & y-1 / y<z \leqq y+1 / y, & \\
& =C y \exp \left(y^{2}\right), & y+1 / y<z,
\end{array}
$$

and for $0 \leqq y \leqq 2$

$$
\begin{aligned}
L(y, z) & =C, & & |y-z|>1, \\
& =C y(1-\log [y|y-z|])+C, & & |y-z| \leqq 1 .
\end{aligned}
$$

In $\S 4$ (3.11) is shown to be less than or equal to $L$; the same is shown for (3.12) in $\S 5$.

That $L$ has the desired monotonicity properties follows by inspection of (3.13) and (3.14). The boundedness of $\int_{-\infty}^{\infty} L(y, z) \exp \left(-z^{2}\right) d z$ is easily seen by estimating the various integrals that arise and using the fact that if $y-1 / y \leqq z \leqq y+1 / y$ and $y \geqq 2$, then $\left|y^{2}-z^{2}\right| \leqq 3$.

$K(x, y, z)$ must be taken equal to

$$
\frac{Q(x, y, z) \exp \left(-z^{2}\right)-Q(x, y, 2 y-z) \exp \left(-(2 y-z)^{2}\right)}{2},
$$

$$
\begin{aligned}
& |y-z|<\min (1,1 /|y|), \\
0, \quad & |y-z| \geqq \min (1,1 /|y|) .
\end{aligned}
$$

Then $K$ clearly has the first two stated properties. The assertion about the total boundedness will be proved in $\S 6$ in the equivalent form of showing that $(z-y) K(x, y, z)$ as a function of $z$ has total variation bounded by a constant independent of $x$ and $y$. Again, since $Q(x, y, z)=-Q(x,-y,-z)$ it will only be necessary to consider the case $y \geqq 0$.

This completes the proof of Lemma 4 except for the three boundedness assertions that are proved in $\$ \$ 4,5$ and 6.

4. Proof that (3.11) is less than or equal to $L$ for $y \geqq 0$. Some useful computational results are given in the next two lemmas. 
LEMMA 5. There is a constant, $C$, such that if $y \geqq 0$ and $y|y-z| \leqq 1$, then

$$
\int_{0}^{1} \frac{1}{1-r} \exp \left(\frac{-(r y-z)^{2}}{1-r^{2}}\right) d r \leqq C[1-\log (y|y-z|)]
$$

and

$$
\int_{0}^{1} \frac{1}{(1-r)^{2}} \exp \left(\frac{-(r y-z)^{2}}{1-r^{2}}\right) d r \leqq \frac{C}{(y-z)^{2}} .
$$

The proofs of these are similar. In each case the integral is divided into integrals over the intersections of $[0,1]$ with $(-\infty, 1-2|y-z| / y],(1-2|y-z| / y, 1-|y-z| / 2 y]$ and $(1-|y-z| / 2 y, 1]$. Since $r y-z=(y-z)-y(1-r), y(1-r) / 2 \leqq|r y-z|$ for the first parts. After replacing $|r y-z|$ by this and $1+r$ by 2 and making the change of variables $u=y^{2}(1-r) / 8$, the estimate is obvious. For the second parts $|y-z| / 2 y$ $\leqq 1-r$; replacing $1-r$ by this and $r y-z$ by 0 will give the estimate. For the third parts $\frac{1}{2}|y-z| \leqq|r y-z|$; replacing $|r y-z|$ by this and $1+r$ by 2 and making the change of variables $u=(y-z)^{2} / 8(1-r)$ will give the desired estimate.

Now define the functions $w(x, y)$ and $p(x, r)$ by

$$
w(x, r)=\left(2^{1 / 2} / \pi\right)\left(\left(1-r^{2}\right) /-\log r\right)^{1 / 2} \exp \left(x^{2} / 2 \log r\right)
$$

and

$$
p(x, r)=\partial w(x, r) / \partial r
$$

LEMMA 6. $\int_{0}^{1}|p(x, r)| d r \leqq 4$.

Let $q(r)=\exp \left(x^{2} / 2 \log r\right)$ and $s(r)=\left(\left(1-r^{2}\right) /-\log r\right)^{1 / 2}$. Then for $0<r<1$, both functions are bounded by 2 and $q$ is decreasing. Differentiating $s$ and using the fact that $-\log r \leqq\left(1-r^{2}\right) / 2 r^{2}$ for $0<r<1$ shows that $s$ is increasing. Using these facts

$$
\int_{0}^{1}|p(x, r)| d r \leqq \frac{2^{1 / 2}}{\pi} \int_{0}^{1}\left|q s^{\prime}+s q^{\prime}\right| d r \leqq \frac{2^{3 / 2}}{\pi} \int_{0}^{1}\left(\left|s^{\prime}\right|+\left|q^{\prime}\right|\right) d r \leqq 4 .
$$

It is now quite easy to estimate (3.11). It may be assumed for this estimation that $|y-z| \leqq \min (1,1 / y)$. Consideration of the cases $y>1$ and $y \leqq 1$ shows that $\left|y^{2}-z^{2}\right|$ $\leqq 3$. Because of this $e^{3} \exp \left(y^{2}-z^{2}\right) \geqq 1$. Multiplying (3.11) by the left side of this last inequality then shows that the absolute value of $(3.12)$ is bounded by

$$
C \exp \left(y^{2}\right)\left|Q(x, y, z) \exp \left(-z^{2}\right)+Q(x, y, 2 y-z) \exp \left(-(2 y-z)^{2}\right)\right| .
$$

Using (4.3) and (3.5), the absolute value part of (4.5) is equal to

$$
\int_{0}^{1}\left[\frac{z-r y}{\left(1-r^{2}\right)^{2}} \exp \left(\frac{-(z-r y)^{2}}{1-r^{2}}\right)+\frac{(2-r) y-z}{\left(1-r^{2}\right)^{2}} \exp \left(\frac{-[y(2-r)-z]^{2}}{1-r^{2}}\right)\right] w(x, r) d r .
$$

In the main parenthesis add

$$
\frac{y(2-r)-z}{\left(1-r^{2}\right)^{2}} \exp \left(\frac{-(z-r y)^{2}}{1-r^{2}}\right)
$$


to the first term and subtract it from the second. Then replacing these pairs of terms by their absolute values and using the fact that $|w(x, r)| \leqq 1$ shows that (4.5) is bounded by the sum of the two following expressions:

$$
C \exp \left(y^{2}\right) \int_{0}^{1} \frac{2 y}{(1-r)(1+r)^{2}} \exp \left(\frac{-(z-r y)^{2}}{1-r^{2}}\right) d r
$$

$$
C \exp \left(y^{2}\right) \int_{0}^{1} \frac{y(1-r)+|y-z|}{\left(1-r^{2}\right)^{2}}\left|1-\exp \left(\frac{4 y(z-y)}{1+r}\right)\right| \exp \left(\frac{-(z-r y)^{2}}{1-r^{2}}\right) d r
$$

Using (4.1), (4.6) is bounded by $C y \exp \left(y^{2}\right)[1-\log (y|y-z|)]$ as desired. Since $y|y-z|<1$, the argument of the first exponential in the integrand of (4.7) is no greater than 4 in absolute value. Therefore, 1 minus this exponential is less than $e^{4}$ times the absolute value of the argument. Consequently, (4.7) is bounded by

$$
C y|z-y| \exp \left(y^{2}\right) \int_{0}^{1} \frac{y(1-r)+|y-z|}{(1-r)^{2}} \exp \left(\frac{-(z-r y)^{2}}{1-r^{2}}\right) d r .
$$

Using (4.1), (4.2) and the fact that $y|y-z| \leqq 1$, shows that (4.8) is also less than $C y \exp \left(y^{2}\right)[1-\log (y|y-z|)]$.

5. Proof that (3.12) is less than or equal to $L$ for $y \geqq 0$. Using (3.5) and the fact that $|w(x, r)| \leqq 1$ shows that

$$
|Q(x, y, z)| \leqq \int_{0}^{1} \frac{|z-r y|}{\left(1-r^{2}\right)^{2}} \exp \left(\frac{-r^{2} y^{2}+2 r y z-r^{2} z^{2}}{1-r^{2}}\right) d r .
$$

It would be sufficient to show that for $|y-z|>\min (1,1 /|y|)$ that $(5.1)$ is bounded by $L$. As will be shown, this can be done in most cases. For $y>2, \frac{1}{2} y<z \leqq y-1 / y$; however, the cancellation produced by the change in sign of $z-r y$ in the definition of $Q$ is essential and the analysis must be more careful. For this case the integral, (3.5), will be split into three parts. Two will be treated in the same manner as the others by replacing $z-r y$ by $|z-r y|$ and $w$ by 1 . The third will be an integral that can be treated directly with an integration by parts.

The proof that (3.12) is less than or equal to $L$ will be done in cases as indicated.

Case 1. $z \leqq \min (0, y-1)$. In this case, if $y \geqq \frac{1}{2}$, the right side of $(5.1)$ is bounded by

$$
\int_{0}^{1} \frac{-z}{(1-r)^{2}} \exp \left(\frac{r y z}{1-r}\right) d r+\int_{0}^{1} \frac{(2-r) r y}{(1-r)^{2}} \exp \left(\frac{-r^{2} y^{2}}{2(1-r)}\right) d r
$$

Both integrations can be performed; both integrals are bounded by $C / y \leqq C$. If $y<\frac{1}{2}$, then $z<-\frac{1}{2}$ and (5.1) is bounded by

$$
\int_{0}^{1} \frac{-2 z(2-r)}{(1-r)^{2}} \exp \left(\frac{-r^{2} z^{2}}{2(1-r)}\right) d r
$$

This can be estimated; it is bounded by $C /-z \leqq C$. 
Case 2. $0<z \leqq \min \left(y-1 / y, \frac{1}{2} y\right)$. This implies that $y \geqq 1$. Multiplying and dividing the right side of (5.1) by $\exp \left(z^{2}\right)$ and replacing $1+r$ by 1 or 2 as required shows that

$$
|Q(x, y, z)| \leqq \exp \left(z^{2}\right) \int_{0}^{1} \frac{|z-r y|}{(1-r)^{2}} \exp \left(\frac{-(z-r y)^{2}}{2(1-r)}\right) d r .
$$

Split the integral on the right side of (5.2) into integrals over $[0,1-(y-z) / 2 y]$ and $[1-(y-z) / 2 y, 1]$. In the first of these $r \leqq \frac{3}{4}$ so that $\frac{1}{4} \leqq 1-r \leqq 1$. Using this to replace $(1-r)$, the first part can then be computed and is bounded by $(C / y) \exp \left(z^{2}\right)$ as desired. For the second integral, using the fact that $z-r y=(z-y)+y(1-r)$ shows that $\frac{1}{2}(y-z) \leqq|z-r y| \leqq 2(y-z)$. Using this and integrating gives the desired estimate for the second integral.

Case 3. $y+1 / y \leqq z \leqq 2 y$. This condition again implies that $y>1$. Multiplying and dividing (5.1) by exp $\left(y^{2}\right)$ and replacing $1+r$ by 1 or 2 as required shows that

$$
|Q(x, y, z)| \leqq \exp \left(y^{2}\right) \int_{0}^{1} \frac{|z-r y|}{(1-r)^{2}} \exp \left(\frac{-(y-r z)^{2}}{2(1-r)}\right) d r
$$

Split this integral into the sum of integrals over

$$
[0,1-2(z-y) / z), \quad[1-2(z-y) / z, 1-(z-y) / 2 z) \text { and }[1-(z-y) / 2 z, 1] \text {. }
$$

For the first part

$$
|z-r y|=|(z-y)+y(1-r)| \leqq 3 y(1-r)
$$

and

$$
|y-r z|=|(y-z)+z(1-r)| \geqq \frac{1}{2} z(1-r) .
$$

Making these substitutions for $|z-r y|$ and $|y-r z|$ and making the substitution $u$ equal to the argument of the exponential shows that this part is bounded by $C y \exp \left(y^{2}\right)$. For the second part use $|z-r y| \leqq 3(z-y)$ and $|y-r z| \geqq 0$ and the estimate is immediate. For the third part use $|z-r y| \leqq 2(z-y)$ and $|y-r z| \geqq \frac{1}{2}(z-y)$ and perform the resulting integration. This gives the desired estimate for the third part.

Case 4. $\max (2 y, y+1) \leqq z$. Split the integral in (5.3) into the sum of integrals over $[0,1-(z-y) / 2 z)$ and $[1-(z-y) / 2 z, 1]$. In the first of these $r \leqq \frac{3}{4}$ so that $\frac{1}{4} \leqq 1-r \leqq 1$. Replacing $1-r$ by the appropriate bound, using $|z-r y| \leqq z$ and making the substitution $u$ equal to the argument of the exponential shows that this part is bounded by $C \exp \left(y^{2}\right)$. For the second, $|z-r y| \leqq z$ and $|y-r z| \geqq \frac{1}{2}(z-y)$. Using these, the resulting integral can be computed directly. This will show that this part is also bounded by $C \exp \left(y^{2}\right)$.

Case 5. $\frac{1}{2} y<z \leqq y-1 / y$. This condition implies that $y \geqq 2^{1 / 2}$. This is the troublesome case and (3.5) will be estimated directly. Let $E$ denote

$$
[1-3(y-z) / 2 y, 1-(y-z) / 2 y]
$$


and

$$
S(r, y, z)=\frac{z-r y}{\left(1-r^{2}\right)^{3 / 2}} \exp \left(\frac{-r^{2} y^{2}+2 r y z-r^{2} z^{2}}{1-r^{2}}\right)
$$

With this notation the following inequalities will be proved:

$$
\left|Q(x, y, z)-\int_{E} \frac{w(x, r)}{\left(1-r^{2}\right)^{1 / 2}} S(r, y, z) d r\right| \leqq \frac{C \exp \left(z^{2}\right)}{\left(y(y-z)^{3}\right)^{1 / 2}}
$$

and

$$
\left|\int_{E} \frac{w(x, r)}{\left(1-r^{2}\right)^{1 / 2}} S(r, y, z) d r\right| \leqq \frac{C \exp \left(z^{2}\right)}{\left(y(y-z)^{3}\right)^{1 / 2}}
$$

These inequalities will give the desired result for this case.

Let $F$ denote the complement of $E$ in $[0,1]$. Using (3.5) and the fact that $|w| \leqq 1$ shows that the left side of (5.4) is bounded by

$$
\int_{F} \frac{|z-r y|}{\left(1-r^{2}\right)^{2}} \exp \left(\frac{-r^{2} y^{2}+2 r y z-r^{2} z^{2}}{1-r^{2}}\right) d r
$$

Multiplying and dividing by $\exp \left(z^{2}\right)$ and replacing $1+r$ by 1 or 2 as appropriate shows that (5.6) and therefore the left side of (5.4) is bounded by

$$
\exp \left(z^{2}\right) \int_{F} \frac{|z-r y|}{(1-r)^{2}} \exp \left(\frac{-(r y-z)^{2}}{2(1-r)}\right) d r .
$$

The set $F$ is the union of the intervals $[0,1-3(y-z) / 2 y)$ and $(1-(y-z) / 2 y, 1]$. On the integral over the first of these, use the fact that $y(1-r) / 3 \leqq|z-r y| \leqq 2 y(1-r)$ and make the substitution $u$ equal to the argument of the exponential. This will show that this part of (5.7) is bounded by

$$
C \exp \left(z^{2}\right) \frac{\exp (y(z-y) / 12)}{(y-z)} .
$$

For the second interval $\frac{1}{2}(y-z) \leqq|z-r y| \leqq 2(y-z)$. Using this and integrating directly again gives the estimate (5.8). Since $y(z-y) \leqq-1$,

$$
\exp (y(z-y) / 12) \leqq C /(y(y-z))^{1 / 2} .
$$

Therefore, (5.8) is bounded by the right side of (5.4) and (5.4) is proved.

Integrating the left side of (5.5) by parts shows that it is bounded by the sum of

$$
\left|\frac{-w(x, r)}{\left(1-r^{2}\right)^{1 / 2}} \int_{r}^{1} S(t, y, z) d t\right|_{E} \mid
$$

and

$$
\left|\int_{E}\left(\left[\frac{d}{d r}\left(\frac{w(x, r)}{\left(1-r^{2}\right)^{1 / 2}}\right)\right] \int_{r}^{1} S(t, y, z) d t\right) d r\right|
$$


To estimate $\int_{r}^{1} S(t, y, z) d t$, let $u=(y-t z) /\left(1-t^{2}\right)^{1 / 2}$. Then

$$
d u=(t y-z) d t /\left(1-t^{2}\right)^{3 / 2}
$$

and

$$
\left|\int_{r}^{1} S(t, y, z) d t\right|=\int_{(y-r z)\left(1-r^{2}\right)^{1 / 2}}^{\infty} \exp \left(y^{2}\right) \exp \left(-u^{2}\right) d u .
$$

Using the fact that for $a>0$,

$$
\int_{a}^{\infty} \exp \left(-u^{2}\right) d u \leqq \frac{C}{a} \exp \left(-a^{2}\right)
$$

the right side of (5.11) is bounded by

$$
C \frac{\left(1-r^{2}\right)^{1 / 2}}{y-r z} \exp \left(y^{2}\right) \exp \left(\frac{-(y-r z)^{2}}{1-r^{2}}\right) \text {. }
$$

Now multiplying and dividing by $\exp \left(z^{2}-y^{2}\right)$ and using the fact that $r \in E$ shows that

$$
\left|\int_{r}^{1} S(t, y, z) d t\right| \leqq \frac{C \exp \left(z^{2}\right)}{(y(y-z))^{1 / 2}} \exp \left(\frac{-(z-r y)^{2} y}{3(y-z)}\right) .
$$

Using (5.12) and the fact that $|w| \leqq 1$ shows that (5.9) is bounded by

$$
\left(C \exp \left(z^{2}\right) /(y-z)\right) \exp (-y(y-z) / 12) \text {. }
$$

This, like (5.8), is bounded by the right side of (5.5).

To estimate (5.10) observe that

$$
\left|\frac{d}{d r}\left(\frac{w(x, r)}{\left(1-r^{2}\right)^{1 / 2}}\right)\right|=\frac{\left(1+x^{2} / \log r\right) \exp \left(x^{2} / 2 \log r\right)}{r 2^{1 / 2} \pi(-\log r)^{3 / 2}} \leqq \frac{C}{r(-\log r)^{3 / 2}} .
$$

Since $1-3(y-z) / 2 y \geqq \frac{1}{4}$,

$$
\frac{C}{r(-\log r)^{3 / 2}} \leqq \frac{C}{(1-r)^{3 / 2}} \text { for } r \in E .
$$

Then using the fact that for $r$ in $E, 1-(y-z) / 2 y \geqq r$, shows that

$$
\left|\frac{d}{d r}\left(\frac{w(x, r)}{\left(1-r^{2}\right)^{1 / 2}}\right)\right| \leqq C\left(\frac{y}{y-z}\right)^{3 / 2}, \quad r \in E .
$$

Using (5.12) and (5.13), (5.10) is bounded by

$$
\int_{E} \frac{C y \exp \left(z^{2}\right)}{(y-z)^{2}} \exp \left(\frac{-y(z-r y)^{2}}{3(y-z)}\right) d r .
$$

Making the substitution $u=(z-r y) \sqrt{ } y /(y-z)^{1 / 2}$ will show that (5.10) is also bounded by the right side of (5.5). This completes the proof for Case 5 .

It is now easy to observe that the five cases considered include all values of $z$ for which $|z-y| \geqq \min (1,1 / y)$ and that in each case (3.12) is bounded by $L$. 
6. The total variation of $(z-y) K(x, y, z)$ for $y \geqq 0$. Observe that the two terms in (3.15) are mirror images of each other around $z=y$. Consequently, when multiplied by $z-y$ they have the same total variation. It is then sufficient to consider the total variation of the function, $H(x, y, z)$, that equals 0 for $|y-z|>m$ and $(y-z) Q(x, y, z) \exp \left(-z^{2}\right)$ for $|y-z| \leqq m$; here and hereafter $m$ will denote $\min (1,1 / y)$.

It is easy to see by inspection of (3.5) and use of the fact that $-r^{2} y^{2}+2 r y z-r^{2} z^{2}$ $=-r^{2}(y-z)^{2}+(1-r) 2 r y z$ that $Q(x, y, z)$ is a differentiable function of $z$ except for $x=0$ and $z=y$. Since for $x>0, H(x, y, y)=0$, the total variation of $H(x, y, z)$ as a function of $z$ for a fixed $x>0$ is less than or equal to twice

$$
\int_{|z-y|<m}\left|\frac{\partial}{\partial z}\left[(y-z) Q(x, y, z) \exp \left(-z^{2}\right)\right]\right| d z .
$$

For the case of $x=0$, observe that by $\S 5$, if $|y-z|=m$, then $|Q(0, y, z)| \leqq L(y, z)$. The definition of $L$ then shows that $H(0, y, z)$ is uniformly bounded for $z=y \pm m$. Therefore, if $H(0, y, y)$ is defined to be 0 , it is clear that the total variation of $H(0, y, z)$ as a function of $z$ is bounded by a constant plus twice (6.1) with $x=0$. Consequently, the desired property of $J$ will be proved by showing that (6.1) is uniformly bounded for $x \geqq 0$ and $y \geqq 0$.

Using (4.4) and (3.5) shows that

$$
Q(x, y, z)=\int_{0}^{1}\left[\frac{z-r y}{\left(1-r^{2}\right)^{2}} \exp \left(\frac{-r^{2} y^{2}+2 r y z-r^{2} z^{2}}{1-r^{2}}\right) \int_{0}^{r} p(x, t) d t\right] d r .
$$

Multiplying by $(y-z) \exp \left(-z^{2}\right)$, differentiating with respect to $z$ and interchanging the order of integration then shows that the integrand in (6.1) is equal to

$$
\left|\int_{0}^{1} p(x, t)\left[\int_{t}^{1}\left(\frac{y(1+r)-2 z}{\left(1-r^{2}\right)^{2}}-\frac{2(z-r y)^{2}(y-z)}{\left(1-r^{2}\right)^{3}}\right) \exp -\left(\frac{(r y-z)^{2}}{1-r^{2}}\right) d r\right] d t\right|
$$

Now it can be readily verified by differentiating that

$$
\begin{aligned}
\frac{y-z}{1-t^{2}} \exp ( & \left.\frac{-(t y-z)^{2}}{1-t^{2}}\right) \\
& +\int_{t}^{1}\left(\frac{2 r(y-z)}{\left(1-r^{2}\right)^{2}}+\frac{2(y-r z)(z-r y)(y-z)}{\left(1-r^{2}\right)^{3}}\right) \exp \left(\frac{-(r y-z)^{2}}{1-r^{2}}\right) d r=0 .
\end{aligned}
$$

Subtracting the left side of this from the inner integral in (6.2) shows that the inner integral equals the sum of

$$
\int_{t}^{1}\left(\frac{y-2 z}{\left(1-r^{2}\right)(1+r)}-\frac{2(y+z)(y-z)(z-r y)}{\left(1-r^{2}\right)^{2}(1+r)}\right) \exp \left(\frac{-(r y-z)^{2}}{1-r^{2}}\right) d r
$$

and

$$
\frac{z-y}{1-t^{2}} \exp \left(\frac{-(t y-z)^{2}}{1-t^{2}}\right)
$$


Writing $z-r y=(z-y)+y(1-r)$ and using (4.1), (4.2) and the fact that $y|y-z| \leqq 1$ shows that (6.3) is bounded by $C(1+y)(1-\log y|y-z|)$. Using Lemma 6 then shows that (6.2) is less than or equal to

$$
C(1+y)(1-\log y|y-z|)+\int_{0}^{1}|p(x, t)| \frac{y-z}{1-t^{2}} \exp \left(\frac{-(t y-z)^{2}}{1-t^{2}}\right) d t .
$$

Since (6.5) is a bound for the integrand in (6.1), showing that the integral of (6.5) with respect to $z$ for $|y-z| \leqq m$ is uniformly bounded for $x \geqq 0$ and $y \geqq 0$ will complete the proof concerning $K$.

The integral of the first term of (6.5) with respect to $z$ for $|y-z| \leqq m$ is easily seen to be uniformly bounded. For the second term write $y-z=y(1-t)+(t y-z)$. The part resulting from having $y(1-t)$ in place of $y-z$ is less than $y$ by Lemma 6 and can be absorbed into the first part of (6.5). The remaining part of the integral is less than or equal to

$$
\int_{0}^{1}|p(x, t)| \frac{|t y-z|}{1-t^{2}} \exp \frac{-(t y-z)^{2}}{1-t^{2}} d t .
$$

To estimate the integral of (6.6) with respect to $z$ for $|y-z| \leqq m$, interchange the order of integration. Performing the inner integration and using Lemma 6 shows that the integral of (6.6) is also uniformly bounded. This completes the proof concerning the total variation of $(z-y) K(x, y, z)$, and therefore completes the proof of Lemma 4.

7. Conjugate function theorems. The principal results concerning conjugate Poisson integrals, $\tilde{f}(x, y)$, and their limit as $x \rightarrow 0^{+}, \tilde{f}(y)$, can now be easily proved.

THEOREM 2. Let $f(y)$ be in $L^{1}\left(\exp \left(-y^{2}\right)\right)$, and for a fixed $y$ let $m$ denote $\min (1,1 /|y|)$. Define

$$
f^{*}(y)=\sup _{u \neq y} \frac{\int_{y}^{u}|f(z)| \exp \left(-z^{2}\right) d z}{\int_{y}^{u} \exp \left(-z^{2}\right) d z}
$$

and let $E_{a}=\left\{y\left|\sup _{x>0}\right| \tilde{f}(x, y) \mid>a\right\}$. Then there exists a constant, $C$, independent of $f$ such that

$$
|\tilde{f}(x, y)| \leqq C\left(\sup _{0<\alpha<\beta<m}\left|\int_{\alpha \leqq|z| \leqq \beta} \frac{f(y-z)}{z} d z\right|+f^{*}(y)\right) \text { for } x>0,
$$

$$
\int_{E_{a}} \exp \left(-y^{2}\right) d y \leqq \frac{C}{a}\|f\|_{1},
$$

$$
\lim _{x \rightarrow 0^{+}} \tilde{f}(x, y)=\tilde{f}(y) \text { exists for almost every } y,
$$

$$
\lim _{\varepsilon \rightarrow 0^{+}} \int_{|y-z|>\varepsilon} f(z) Q(0, y, z) \exp \left(-z^{2}\right) d z \text { exists for almost every } y,
$$

the limits in (c) and (d) are equal for almost every $y$. 
Using Lemma $4, \tilde{f}(x, y)$ may be written as the sums of

$$
\int_{-\infty}^{\infty} J(x, y, z) \exp \left(-z^{2}\right) f(z) d z
$$

and

$$
\int_{y-m}^{y+m} K(x, y, z) f(z) d z
$$

Corollary 2 of [2] then shows that (7.2) is bounded by $C f^{*}(y)$. By use of Lemma 1, (7.3) is clearly bounded by the first term on the right side of (a).

Lemma 1 of [2] shows immediately that (7.2) satisfies conclusion (b). To apply Lemma 3 to (7.3), a suitable partition of $(-\infty, \infty)$ must be defined. For this, use the interval $[0,2]$, intervals of length $2^{-n+1}$ between $2^{n}$ and $2^{n+1}, n=1,2, \ldots$, and the mirror images of these intervals for negative numbers. This subdivision clearly satisfies conditions 1 and 2 of the definition of a partition with property $A$. Now, on $[0,2], \exp \left(-x^{2}\right)$ changes by a factor of $e^{4}$. If $y$ is the left end of one of the other subintervals of $[0, \infty)$, the length of the interval is less than $4 / y$ and $\exp \left(-x^{2}\right)$ changes by a factor less than or equal to

$$
\exp \left(-y^{2}+(y+4 / y)^{2}\right)=\exp \left(8+16 y^{-2}\right) \leqq e^{12} .
$$

By symmetry, on the subintervals of $(-\infty, 0]$, exp $\left(-x^{2}\right)$ changes by no more than the factor $e^{12}$. Consequently, this partition has property $A$. Furthermore, if $y$ is in one of the intervals, $m=\min (1,1 /|y|)$ is not greater than half the length of the interval. Consequently, Lemma 3 proves (b) for (7.3). Since the sum of two functions with the property (b) also has that property, conclusion (b) is proved.

Conclusion (c) is certainly true for $f$ a polynomial since, by Theorem $1, \tilde{f}(x, y)$ is then the polynomial (3.6). Conclusion (c) follows in the usual way from this, conclusion (b) and the fact that polynomials are dense in $L^{1}\left(\exp \left(-y^{2}\right)\right)$.

To prove (d), let $\tilde{f}_{\varepsilon}(y)$ denote the integral appearing in $(\mathrm{d})$. Then $\tilde{f}_{\varepsilon}(y)$ is the sum of

$$
\int_{|y-z|>\varepsilon} J(0, y, z) f(z) \exp \left(-z^{2}\right) d z
$$

and

$$
\int_{\varepsilon<h<m}\left(\frac{f(y+h)-f(y-h)}{h}\right) h K(0, y, y+h) d h .
$$

If $f$ is a polynomial, (7.4) and (7.5) clearly converge as $\varepsilon \rightarrow 0^{+}$because of Lemma 4 and the dominated convergence theorem. Again, because of Lemma 4, the method used to prove parts (a) and (b) will give the same results for $f_{\varepsilon}(y)$. Part (d) then is proved in the same manner as part (c). That $\tilde{f}_{\varepsilon}(y)$ and $\tilde{f}(x, y)$ converge to the same function is clear for polynomials by writing $\tilde{f}(x, y)$ also as the sum of integrals 
using $J$ and $K$. The dominated convergence theorem shows that these two integrals and (7.4) and (7.5) converge respectively to the expressions (7.4) and (7.5) with $\varepsilon$ replaced by 0 . Part (b) and the analogy for $f_{\varepsilon}(y)$ then prove that the limits in (c) and (d) are equal almost everywhere for any function since polynomials are dense in $L^{1}\left(\exp \left(-y^{2}\right)\right)$.

THEOREM 3. Let $f(y)$ be in $L^{p}\left(\exp \left(-y^{2}\right)\right), 1<p<\infty$, let $x$ be greater than 0 , and let $R(x, y, z)$ be the Poisson kernel defined in (3.2). Then there exist constants, $A_{p}$, depending only on $p$ such that

(a) $\lim _{x \rightarrow 0^{+}}\left\|f(x, y)-f^{\prime}(y)\right\|_{p}=0$,

(b) $\|\tilde{f}(y)\|_{p} \leqq A_{p}\|f\|_{p}$,

(c) $f^{\prime}(x, y)=\int_{-\infty}^{\infty} \tilde{f}(z) R(x, y, z) \exp \left(-z^{2}\right) d z$ for almost every $y$,

(d) $\left\|\sup _{x>0}|\tilde{f}(x, y)|\right\|_{p} \leqq A_{p}\|\tilde{f}(y)\|_{p}$

(e) $\left\|f^{\prime}(x, y)\right\|_{p} \leqq\left\|f^{f}(y)\right\|_{p}$ for all $x>0$,

(f) $f(y)$ has Hermite expansion $\sum a_{n}(2 n)^{1 / 2} H_{n-1}(y)$ where $a_{n}$ is the nth coefficient inf's Hermite expansion.

First, by part (a) of Theorem 2, Lemma 1 of [2] and Lemma 2 with the partition used to prove Theorem 2, there exists for $1<p<\infty$ constants $A_{p}$ such that

$$
\left\|\sup _{x>0}|\tilde{f}(x, y)|\right\|_{p} \leqq A_{p}\|f\|_{p} .
$$

Part (a) follows from this, part (c) of Theorem 2 and the dominated convergence theorem. Part (b) then follows from part (a) and (7.6).

Part (c) is obvious for polynomials by use of Corollary 3 of [2] and Theorem 1. If $1<p \leqq 2$ and $f$ is in $L^{p}\left(\exp \left(-y^{2}\right)\right)$, then a polynomial, $g$, may be chosen to make $\|f-g\|_{p}$ arbitrarily small; this follows from the fact that polynomials are dense in $L^{2}\left(e^{-y^{2}}\right)$. Then,

$$
\left\|f(x, y)-\int_{-\infty}^{\infty} f(z) R(x, y, z) \exp \left(-z^{2}\right) d z\right\|_{p}
$$

is less than or equal to

$$
\|\tilde{f}(x, y)-\tilde{g}(x, y)\|_{p}+\left\|\int_{-\infty}^{\infty}[\tilde{g}(z)-\tilde{f}(z)] R(x, y, z) \exp \left(-z^{2}\right) d z\right\|_{p} .
$$

Now (7.6) can be used on the first term of (7.8) and Corollary 3 of [2] followed by part (b) on the second term. This shows that (7.8) can be made arbitrarily small and that, therefore, (7.7) equals 0 . This proves part (c) for $1<p \leqq 2$. If $p \geqq 2, f$ is also in $L^{2}$ and (c) is, therefore, valid in this case also.

Parts (d) and (e) follow immediately from (c) and Corollary 3 of [2]. Part (f) is a consequence of Theorem 1 and part (a).

Finally a substitute result can be stated in case $p=1$. 
THEOREM 4. If

$$
\int_{-\infty}^{\infty}|f(y)|\left(\log ^{+}|f(y)|\right) \exp \left(-y^{2}\right) d y=A
$$

is finite, then there exists a constant, $C$, such that

(a) $\lim _{x \rightarrow 0^{+}}\|f(x, y)-\tilde{f}(y)\|_{1}=0$,

(b) $\left\|f^{f}(y)\right\|_{1} \leqq C(1+A)$,

(c) $f(x, y)=\int_{-\infty}^{\infty} f(z) R(x, y, z) \exp \left(-z^{2}\right) d z$ for almost every $y$,

(d) $\left\|\sup _{x>0} \tilde{f}(x, y)\right\|_{1} \leqq C(1+A)$,

(e) $\|f(x, y)\|_{1} \leqq\|\tilde{f}(y)\|_{1}$ for $x>0$.

Part (d) follows immediately by use of part (b) of Theorem 2, parts (d) and (b) of Theorem 3, and Theorem (4.34), Vol. II, p. 118 of [6]. Part (a) follows from this and the pointwise convergence proved in part (c) of Theorem 2. Part (b) is immediate from (a) and (d).

To prove part (c) let $f_{N}(y)$ be the function equal to $f(y)$ where $|f(y)| \leqq N$ and 0 elsewhere. By Theorem 3 part (c), part (c) is true for $f_{N}$ and therefore

is bounded by

$$
\left\|f(x, y)-\int_{-\infty}^{\infty} f(z) R(x, y, z) \exp \left(-z^{2}\right) d z\right\|_{1}
$$

$$
\left\|\tilde{f}(x, y)-\tilde{f}_{N}(x, y)\right\|_{1}+\left\|\int_{-\infty}^{\infty}\left(\tilde{f}_{N}(z)-f(z)\right) R(x, y, z) \exp \left(-z^{2}\right) d z\right\|_{1} .
$$

Unfortunately (b), (d) and Corollary 3 of [2] applied to (7.10) will only show that it is less than a constant. To show that (7.9) is zero, let $h(y)=f(y)-f_{N}(y)$ and let

$$
a=\int_{-\infty}^{\infty}|h|\left(1+\log ^{+}|h|\right) \exp \left(-y^{2}\right) d y .
$$

If $a<1$, apply (b) to $h(y) / a$ and use the fact that

$$
\log ^{+}(|h| / a) \leqq\left(1+\log ^{+}|h|\right)\left(1+\log ^{+}(1 / a)\right) \text {. }
$$

This produces the inequality $\|\tilde{h}\|_{1}=a\|\tilde{h} / a\|_{1} \leqq C a\left(1+\log ^{+}(1 / a)\right)$. The same applies to $\left\|\sup _{x>0}|\tilde{h}(x, y)|\right\|_{1}$. Since as $N \rightarrow \infty, a \rightarrow 0$, these inequalities and Corollary 3 of [2] show that (7.10) can be made arbitrarily small and that (7.9) is 0 . This completes the proof of (c).

Part (e) is immediate from (c) and Corollary 3 of [2].

Conclusion (f) of Theorem 3 is false here since a function satisfying the hypotheses of Theorem 4 need not have an Hermite expansion. A simple example of such a function is $\exp \left(y^{2}\right) /\left(1+y^{4}\right)$.

\section{REFERENCES}

1. A. P. Calderon and A. Zygmund, On the existence of certain singular integrals, Acta Math. 88 (1952), 85-139.

2. B. Muckenhoupt, Poisson integrals for Hermite and Laguerre expansions, Trans. Amer. Math. Soc. 139 (1969), 231-242 
3. B. Muckenhoupt and E. M. Stein, Classical expansions and their relation to conjugate harmonic functions, Trans. Amer. Math. Soc. 118 (1965), 17-92.

4. E. M. Stein, On limits of sequences of operators, Ann. of Math. 74 (1961), 140-170.

5. G. Szegö, Orthogonal polynomials, Amer. Math. Soc. Colloq. Publ. Vol. 23, Amer. Math. Soc., Providence, R. I., 1959.

6. A. Zygmund, Trigonometric series, Vols. I, II, 2nd ed., Cambridge Univ. Press, New York, 1959.

RUtgers, The State University,

NeW BRUNSWICK, NEW JERSEY 